\title{
Changing indications of prenatal diagnosis in molecular era: experience of single centre in North India
}

\author{
Aradhana Dwivedi ${ }^{1}$, Reema Kumar Bhatt ${ }^{2}$, Kamal Kishore ${ }^{3 *}$
}

\begin{abstract}
${ }^{1}$ Department of Pediatrics, Division of Clinical Genetics, ${ }^{2}$ Department of Obstetrics and Gynecology, Division of Fetal Medicine, ${ }^{3}$ Department of Paediatric Surgery, Army Hospital Research, Delhi Cantt, New Delhi, India
\end{abstract}

Received: 26 August 2021

Accepted: 08 September 2021

\section{*Correspondence:}

Dr. Kamal Kishore,

E-mail: bhatt.kamal.k@gmail.com

Copyright: ( $)$ the author(s), publisher and licensee Medip Academy. This is an open-access article distributed under the terms of the Creative Commons Attribution Non-Commercial License, which permits unrestricted non-commercial use, distribution, and reproduction in any medium, provided the original work is properly cited.

\section{ABSTRACT}

Background: About 3-5\% of pregnancies are complicated by chromosomal aberrations and birth defects. In the past prenatal genetic testing is identified to be largely restricted to the detection of chromosomal abnormalities like aneuploidy. With advances in the field of medical genetics, there has been substantial rise in use of prenatal genetic testing and we wanted to find out the same in our cohort of patients.

Methods: Study design was retrospective, single-center observational study. Pregnant patients who underwent invasive testing for prenatal genetic disorder at fetal medicine specialty were considered for inclusion in the study. The invasive procedures that were performed in our study were amniocentesis, chorionic villous sampling (CVS) and cordocentesis. Results: Total 515 pregnant women underwent prenatal testing. Amniocentesis was the most common procedure to be performed accounting for about $74 \%$ of total cases. In our cohort, abnormal aneuploidy screening was the most common indication for performing prenatal diagnosis (64\% of cases), while $12.8 \%$ underwent prenatal diagnosis due to abnormalities/genetic disorder in previous child. Abnormalities in antenatal ultrasonography accounted for $16 \%$ of cases. Quantitative fluorescene polymerase chain reaction, rapid aneuploidy testing (QFPCR) was performed in all the cases. Karyotype was performed in 273 cases while chromosomal microarray was performed on 92 samples. Multiplex ligation dependent probe amplification (MLPA) was done for 15 patients. Targeted mutation testing (Sanger sequencing) was done on 121 prenatal samples. Exome sequencing was performed on 14 fetuses. Out of 515 a total of 79 fetuses (15.3\%) were found to have genetic disorder. Aneuploidies were identified in 11 fetuses (2.1\%), 12 fetuses (2.3\%) were found to have pathogenic CNVs (Copy number variants). Single gene disorders were found in 56 fetuses $(10.8 \%)$.

Conclusions: We have moved very rapidly from Karyotyping to chromosomal microarray to exome sequencing. There has been rapid change in the indications for prenatal Diagnosis from yesteryears with coming of new era of genetics.

Keywords: Prenatal genetic testing, Rapid aneuploidy testing, Karyotyping, Chromosomal microarray, Sanger sequencing, Exome sequencing

\section{INTRODUCTION}

About $3-5 \%$ of pregnancies are complicated by chromosomal aberrations and birth defects. In the past prenatal genetic testing is identified to be largely restricted to the detection of chromosomal abnormalities like aneuploidy. ${ }^{1}$ With advances in the field of medical genetics, there has been substantial rise in use of prenatal genetic testing. Compared to conventional tests like karyotyping and fluorescence in-situ hybridization (FISH), use of modern tests (example- chromosomal microarray, sanger sequencing, next generation sequencing) allow rapid diagnosis of specific disorders with a greater sensitivity. ${ }^{2-4}$ With increasing and safer use of invasive 
prenatal techniques coupled with availability of modern genetic tests, the indications of prenatal genetic screening have widened. Moving from advanced maternal age as indication for screening to prenatal chromosomal abnormalities, past pregnancies with aneuploidy or a child born with genetic disorder, such indications will be more diverse in future with use of whole-exome and wholegenome sequencing. ${ }^{4}$

In India, high birth rate with growing population size is paralleled by increasing rate of genetic disorders and congenital malformations in pregnancies. Nearly two decades ago, a half-million foetuses and over 20,000 pregnancies were diagnosed with genetic disorders and Down's syndrome respectively. ${ }^{5,6}$ This represents the significant burden of the genetic disorders in Indian setting necessitating wider use of prenatal testing. With availability of a variety of new and advanced techniques, increasing number of disorders are being diagnosed. As one of the expert center in offering prenatal genetic testing, we assessed indications and genetic disorders identified after prenatal genetic testing at our center from North India.

\section{METHODS}

\section{Study setting}

This study was conducted at a tertiary care center providing speciality services in fetal medicine, Medical genetics and Pediatric Surgery. The center caters to urban and semi-urban population. It is equipped with facilities for modern prenatal genetic tests.

\section{Study design}

The study was retrospective, single-center observational study.

\section{Study duration}

The data of patients who were referred to this hospital for antenatally detected congenital anomalies and who underwent prenatal genetic testing between January 2015 to December 2018 was screened for selection of study participants.

\section{Study population}

Pregnant patients who underwent invasive testing for prenatal genetic disorder at fetal medicine specialty were considered for inclusion in the study.

\section{Study procedures}

The invasive procedures that were performed in our study were amniocentesis, chorionic villous sampling (CVS) and cordocentesis. These procedures have been described in detail in the literature. ${ }^{7-9}$ A brief description of procedures performed at our center is below.

\section{Amniocentesis}

Under aseptic precautions and ultrasound-guidance, amniocentesis was performed using a 22-gauge needle inserted transabdominally by the expert fetal medicine specialist. In all cases, procedure was performed after 12 weeks of gestation. As a routine protocol at our center, cord and placenta are avoided during needle puncture. Nearly 20-ml of fluid was collected form the largest pocket whenever possible in sterile amnio collection tubes which were immediately refrigerated at $2-8{ }^{\circ} \mathrm{C}$. In case of failed attempt, second procedure was attempted at least 8-10 days after the first procedure.

\section{CVS}

Under aseptic precautions and ultrasound-guidance, CVS was performed using either transabdominal or transvaginal route and was carried out after 10-week of gestation in all cases. CVS biopsy needle of 17-20 gauge was pierced transabdominally under local anaesthesia. Biopsy forceps with plastic stylet with syringe aspiration was done when using transvaginal route. Nearly $5 \mathrm{mg}$ of villi were ensured in each sample based on visual impression of the expert. Sample was transferred to sterile container was stored at $2-8^{0} \mathrm{C}$.

\section{Cordocentesis}

Under aseptic precautions and ultrasound-guidance, a 22gauge needle was inserted transabdominally into the umbilical vein. A saline flush confirmation was used to check for the correct placement of the needle. In patients who had anterior placenta, cord puncture at the insertion site was performed. Blood aspirated in the syringe. One $\mathrm{ml}$ of this blood was checked by automatic blood analyser for mean corpuscular haemoglobin. In all cases, the procedure was performed with completed 18 -weeks of gestation.

After any procedure, patients were asked to take complete bed rest for at least 24 hours. In high-risk cases, postprocedure antibiotic prophylaxis (amoxicillin $500 \mathrm{mg}$ three times daily) was given for 3 days. In cases of pain at injection site, analgesics (acetaminophen) were administered on as and when required basis.

\section{Genetic tests}

A special genetics lab at our center performed all the genetic tests. These included Karyotype, Quantitative Fluoroscence Polymerase Chain Reaction (QFPCR), Multiplex Ligation dependent probe amplification (MLPA), Chromosomal microarray (CMA), Sanger sequencing and next generation sequencing based test (clinical exome, whole exome sequencing).

\section{Data capture and analysis}

The data on indications for genetic testing and specific disorder identified was entered into the Microsoft excel 
sheet. Data was analysed using descriptive statistics. Frequency and percentage was used to present the categorical data and mean and standard deviation to present the continuous data.

\section{RESULTS}

In a study period of 4 years, total 515 pregnant women underwent prenatal testing. Baseline characteristics are recorded in table 1.
Most women were second gravida in the age group of 31 to 35 years. Most of the prenatal diagnostic procedures were performed between 12 to 20 weeks of gestation (Table 1) keeping in mind the safety and Medical Termination of Pregnancy (MTP) act of country. Amniocentesis was the most common procedure to be performed accounting for about $74 \%$ of total (Figure 1).

Table 1: Baseline characteristics.

\begin{tabular}{|c|c|c|c|c|c|c|c|c|c|}
\hline \multirow[b]{2}{*}{ Parameter } & \multicolumn{5}{|c|}{ Age (years) } & \multicolumn{4}{|l|}{ Gravida } \\
\hline & $\leq 25$ & $26-30$ & $31-35$ & $\geq 36$ & Mean \pm SD & $\begin{array}{l}\text { Primi } \\
\text { (First) }\end{array}$ & Second & Third & $\begin{array}{l}\text { Four or } \\
\text { more }\end{array}$ \\
\hline \multirow[t]{3}{*}{$\begin{array}{l}\text { Observations } \\
(\%)\end{array}$} & $\begin{array}{l}22 \\
(4.3)\end{array}$ & $\begin{array}{l}248 \\
(48.1)\end{array}$ & $\begin{array}{l}185 \\
(35.9)\end{array}$ & $\begin{array}{l}60 \\
(11.6)\end{array}$ & $31.2 \pm 3.7$ & $\begin{array}{l}114 \\
(22.1)\end{array}$ & $278(54)$ & $108(21)$ & $15(2.9)$ \\
\hline & \multicolumn{5}{|c|}{ Parity } & \multicolumn{4}{|c|}{ Previous abortions } \\
\hline & None & & One & \multicolumn{2}{|l|}{ Two } & None & $1-2$ & \multicolumn{2}{|l|}{$\geq 3$} \\
\hline \multirow[t]{3}{*}{$\begin{array}{l}\text { Observations } \\
(\%)\end{array}$} & \multicolumn{2}{|c|}{$192(37.3)$} & $260(50.5)$ & \multicolumn{2}{|c|}{$63(12.2)$} & $363(70.5)$ & $\begin{array}{l}147 \\
(28.5)\end{array}$ & \multicolumn{2}{|l|}{$5(1)$} \\
\hline & \multicolumn{9}{|c|}{ Period of gestation (Weeks) } \\
\hline & \multicolumn{3}{|r|}{$12.1-20$} & \multicolumn{2}{|c|}{$20.1-24$} & \multicolumn{2}{|l|}{$\geq 24$} & \multicolumn{2}{|l|}{ Mean \pm SD } \\
\hline $\begin{array}{l}\text { Observations } \\
(\%)\end{array}$ & \multicolumn{2}{|l|}{$55(10.7)$} & 397 (77.9) & \multicolumn{2}{|l|}{$48(9.3)$} & \multicolumn{2}{|l|}{$15(2.9)$} & \multicolumn{2}{|l|}{$18 \pm 3.4$} \\
\hline
\end{tabular}

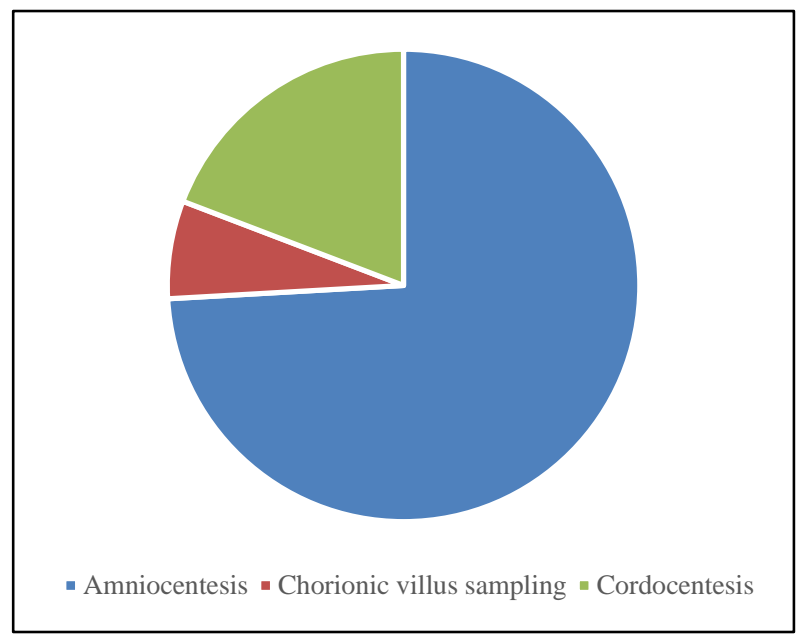

Figure 1: Types of procedures.

In our cohort, abnormal aneuploidy screening was the most common indication for performing prenatal diagnosis (64\% of cases), while $12.8 \%$ underwent prenatal diagnosis due to abnormalities/genetic disorder in previous child. Abnormalities in antenatal ultrasonography accounted for $16 \%$ of cases (Figure 2). QFPCR was performed in all the cases. Karyotype was performed in 273 cases while chromosomal microarray was performed on 92 samples. MLPA was done for 15 patients. Targeted mutation testing (Sanger sequencing) was done on 121 prenatal samples. Exome sequencing was performed on 14 fetuses (Figure $3)$.

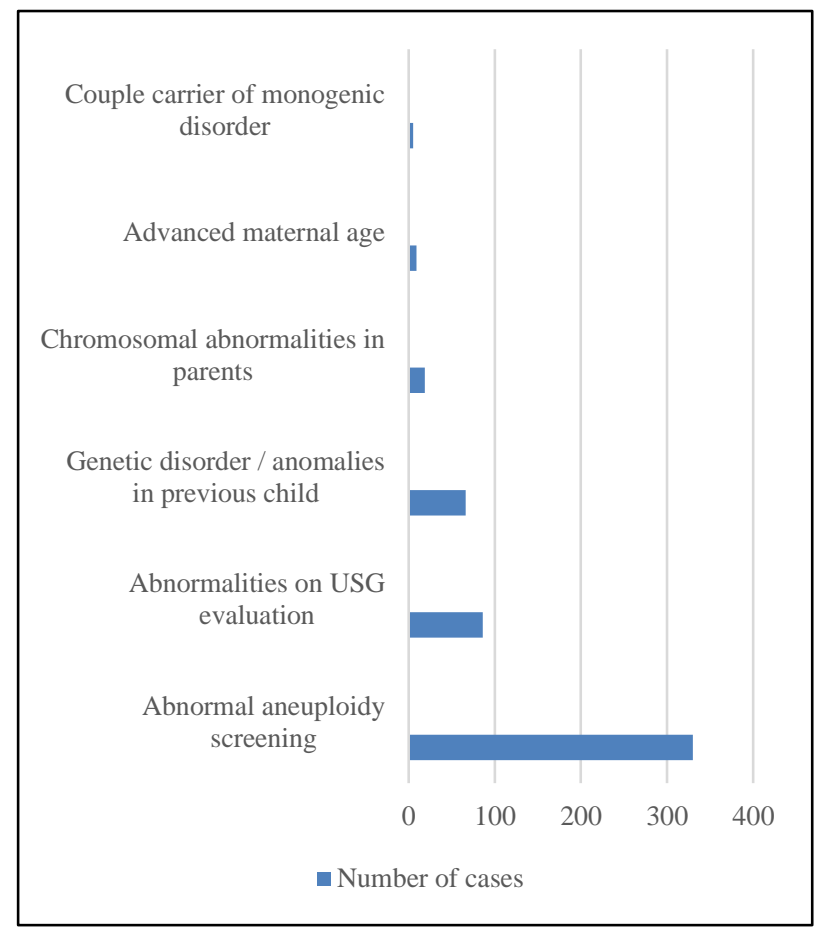

Figure 2: Indications for performing genetic testing.

Out of 515 a total of 79 fetuses $(15.3 \%)$ were found to have genetic disorder (Figure 4). Aneuploidies were identified in 11 fetuses $(2.1 \%), 12$ fetuses $(2.3 \%)$ were found to have pathogenic CNVs (Copy number variants). Single gene disorders were found in 56 fetuses $(10.8 \%$ ) (Figure 5). 


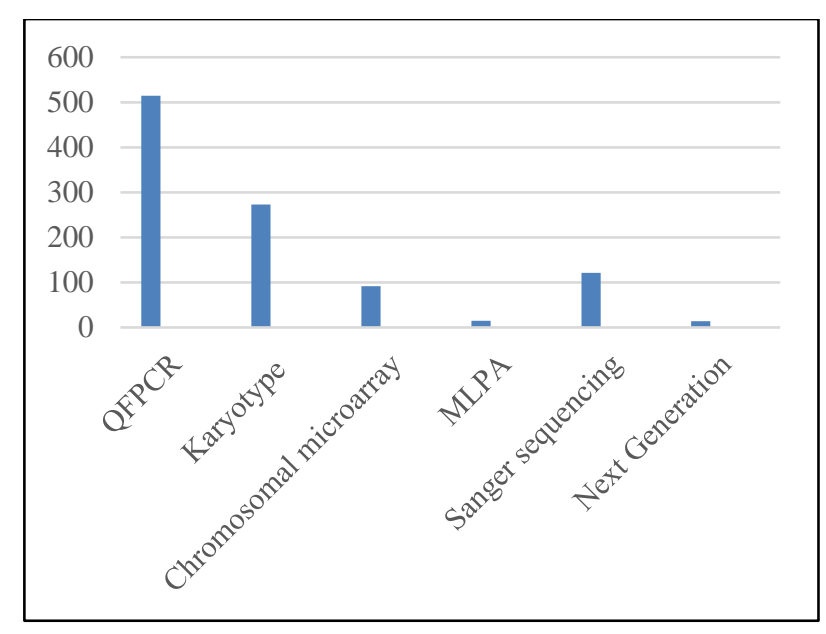

Figure 3: Types of genetic tests performed.

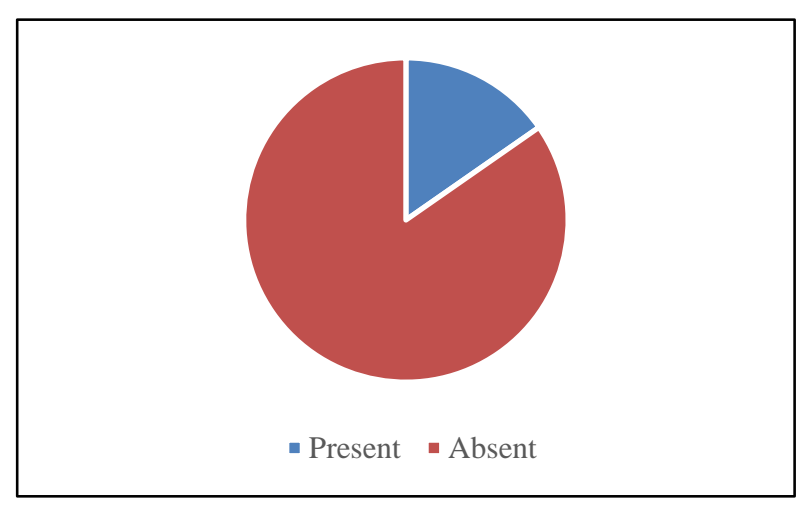

Figure 4: Genetic disorders identified in fetuses.

\section{DISCUSSION}

Over the last few decades, genetic diagnostic techniques have advanced dramatically. Application of these newer techniques into prenatal diagnosis has been transformational. Use of chromosomal microarray and next generation sequencing based tests in genetic clinics helps in establishing the genotype of proband, thereby enabling prognosis, accurate genetic counselling including risk of recurrence in subsequent pregnancies.

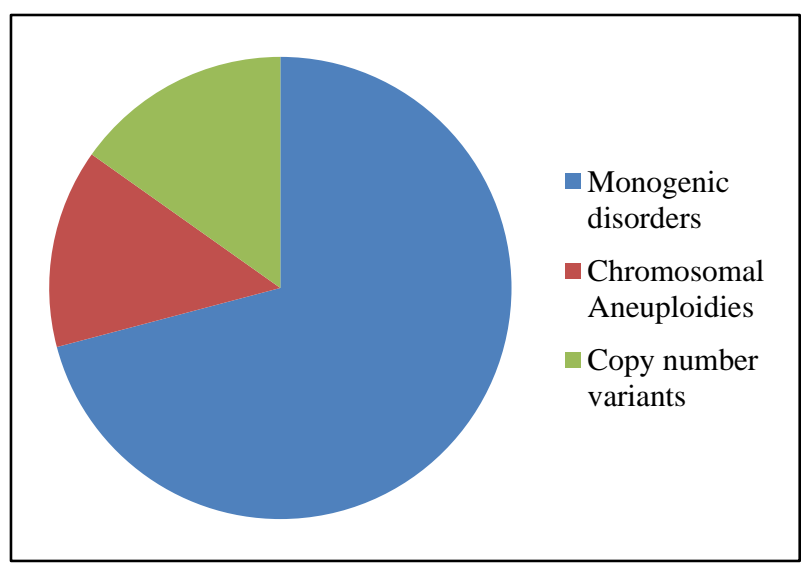

Figure 5: Types of genetic disorders identified.
In our cohort, most common indication of performing prenatal testing was abnormal aneuploidy screening results in first or second trimester due to universal application of biochemical screening withstanding ACOG recommendations..$^{10}$ QFPCR was done for all the foetuses for rapid screening of common aneuploidies of chromosome $21,18,13$ and sex chromosome. QF-PCR is based on the amplification of chromosome-specific DNA sequences (STR, short tandem repeats) polymorphic in length between subjects.

The amplified segments can be visualized by means of fluorescent primers, and quantified as peak areas on automated DNA scanners. Normal heterozygous subjects are expected to show two peak areas (peaks ratio 1:1) for each chromosome analysed, while trisomies are visualized either as an extra peak (triallelic subjects) or as a 2:1 ratio peak between the two areas. ${ }^{11}$ MLPA was done on 15 samples with history of DMD (Duchenne muscular dystrophy) and SMA (Spinal muscular atrophy) in probands. MLPA is a multiplex PCR method detecting copy numbers of up to 60 different genomic DNA sequences. It is the test of choice for conditions like DMD and SMA in which exonic deletions are most common pathogenic variant. We ordered Chromosomal microarray analysis (CMA) if fetus was found to have one major anomaly or multiple minor anomalies not conforming to monogenic disorder. CMA detects copy number variants (submicroscopic deletions/duplications) which are undetectable by conventional karyotype. The yield of CMA in our cohort was $13 \%$ (12/92). Wapner et al demonstrated that in pregnancies with fetal structural anomalies and a normal karyotype there was an incremental diagnostic yield of about $6 \% .^{12}$ Additional diagnostic yield of CMA over karyotype in our study was $8.3 \%$. Targeted mutation analysis by sanger sequencing was performed on 121 fetuses with family history of affected sibling with genetic disorder or couple found to be carrier of mendelian disorder. Genetic diagnosis in proband could be established with involvement of clinical geneticist and application of newer next generation sequencing based genetic tests. Underlying genetic disorder was uncovered in few patients wrongly labelled as cerebral palsy, by doing good phenotyping and genetic testing. Accurate molecular diagnosis in proband enabled us to predict risk of recurrence and prenatal diagnosis in subsequent pregnancies.

In our cohort exome sequencing was performed on 14 anomalous fetuses with negative microarray results. It didn't offer any additional diagnostic yield; however, this is too small sample size to infer. Next generation sequencing has been widely applied in prenatal genomics. Exome (coding part of genome) sequencing can detect single nucleotide variants and small duplications or deletions giving additional yield over chromosomal microarray and karyotype. The International Society for Prenatal Diagnosis (ISPD), the Society for Maternal Fetal Medicine (SMFM), and the Perinatal Quality Foundation (PQF) has issued joint position statement for considering 
sequencing in prenatal period. In one of the biggest studies on prenatal exomes by Lord et al on cohort of 610 fetusparent trios, diagnostic yield of $8.5 \%$ of overall and in $15.4 \%$ of fetuses with more than one anomaly is reported. ${ }^{13}$ (With genetic testing increasing in leaps and bounds, exome sequencing in prenatal samples may become the standard of care in future.)

\section{CONCLUSION}

In the yesteryears when advanced maternal age was the main indication of prenatal testing it now forms a small cohort in our study, this is attributable to biochemical screening in first trimester becoming standard of care. The revolution in genetics has paved way for an array of prenatal tests which have been added in the armamentarium and has brought about a change in indications for prenatal diagnosis.

Funding: No funding sources Conflict of interest: None declared

Ethical approval: The study was approved by the Institutional Ethics Committee

\section{REFERENCES}

1. Hixson L, Goel S, Schuber P, Faltas V, Lee J, Narayakkadan A, et al. An overview on prenatal screening for chromosomal aberrations. J Lab Autom. 2015;20:562-73.

2. Larrabee PB, Johnson KL, Pestova E, Lucas M, Wilber K, LeShane ES, et al. Microarray analysis of cell-free fetal DNA in amniotic fluid: a prenatal molecular karyotype. Am J Hum Genet. 2004;75:48591.

3. Dugoff L, Norton ME, Kuller JA. The use of chromosomal microarray for prenatal diagnosis. Am J Obstet Gynecol. 2016;215(4):B2-9.

4. Norton ME, Rink BD. Changing indications for invasive testing in an era of improved screening. Semin Perinatol. 2016;40:56-66.
5. Verma IC. Burden of genetic disorders in India. Indian J Pediatr. 2000;67(12):893-8.

6. Verma IC, Bijarnia S. The burden of genetic disorders in India and a framework for community control. Community Genet. 2002;5(3):192-6.

7. Ghi T, Sotiriadis A, Calda P, Da Silva Costa F, RaineFenning N, Alfirevic Z, et al. International Society of Ultrasound in Obstetrics and Gynecology (ISUOG). ISUOG Practice Guidelines: invasive procedures for prenatal diagnosis. Ultrasound in Obstetrics \& Gynecology. 2016;48:256-68.

8. Alfirevic Z, Navaratnam $K$, Mujezinovic $F$. Amniocentesis and chorionic villus sampling for prenatal diagnosis. Cochrane Database of Systematic Reviews. 2017;9:CD003252.

9. Shim SS. Chorionic villus sampling. J Genet Med. 2014;11:43-8.

10. ACOG Practice Bulletin No. 163: screening for fetal chromosomal abnormalities. Obstet Gynecol. 2016;127:123-37.

11. Adinolfi M, Pertl B, Sherlock J. Rapid detection of aneuploidies by microsatellite and the quantitative fluorescent polymerase chain reaction. Prenat Diagn. 1991;17:1299-311.

12. Wapner RJ, Martin CL, Levy B. Chromosomal microarray versus karyotyping for prenatal diagnosis. N Engl J Med. 2012;367(23):2175-84.

13. Lord J, McMullan DJ, Eberhardt RY. Prenatal exome sequencing analysis in fetal structural anomalies detected by ultrasonography (PAGE): a cohort study. Lancet. 2019;393:747-57.

Cite this article as: Dwivedi A, Bhatt RK, Kishore $\mathrm{K}$. Changing indications of prenatal diagnosis in molecular era: experience of single centre in North India. Int J Reprod Contracept Obstet Gynecol 2021;10:3744-8. 